\title{
Identification of Pile-up Using the Quality Factor of Pulse Shapes in the ATLAS Tile Calorimeter
}

\author{
Christophe Clement, Pawel Klimek \\ (on behalf of the ATLAS Collaboration)
}

\begin{abstract}
The ATLAS experiment records data from the proton-proton collisions produced by the Large Hadron Collider (LHC). The Tile Calorimeter is the hadronic sampling calorimeter of ATLAS in the region $|\eta|<1.7$. It uses iron absorbers and scintillators as active material. The LHC will provide collisions every $25 \mathrm{~ns}$, putting very strong requirements on the energy measurement in presence of energy deposits from different collisions in the same read out window and physical calorimeter channel (pile-up). In 2011 the LHC is running with filled bunches at 50 ns spacing and at intensities which yield up to about 8 proton-proton collisions per bunch crossing. We present a quality factor that can be computed online for each collision and for each calorimeter channel within the $10 \mu s$ latency of the ATLAS first level trigger (L1 trigger), and could allow to identify calorimeter channels presenting pile-up. In presence of a poor quality factor the data from the corresponding channel is read out with additional information to allow for an offline dedicated treatment of the signals to account for pile-up.
\end{abstract}

\section{INTRODUCTION}

$\mathbf{T}$ HE Large Hadron Collider (LHC), currently under operation at CERN, with its unprecedented high energy and luminosity extends the frontier of particle physics. Bunches of up to $10^{11}$ protons collide 40 million times per second at a center of mass energy of $7 \mathrm{TeV}$ and a design luminosity of $10^{34} \mathrm{~cm}^{-2} s-1$. Nominally the LHC will operate with proton bunches crossing every $25 \mathrm{~ns}$, although in 2011 it has operated with 50 ns bunch spacing and with an expected average number of 8 proton-proton collisions per bunch crossing. The high interaction rates, energies, particle multiplicities, radiation doses and need for precision measurements require new standards for the design of particle detectors at LHC. ATLAS [1] is one of the major experiments designed to exploit the proton-proton data in this stringent environment.

ATLAS is a general-purpose experiment, whose goal is to cover a broad range of experimental particle physics phenomena, with the long sought Higgs boson [2] and supersymmetry [3] on top of the wanted list. The ATLAS experiment needs to be sensitive to a large number of possible decay channels and so must provide an excellent particle identification and high resolution measurements of energies, momenta and directions for the outgoing particles in the proton-proton collisions.

Manuscript received November 15, 2011.

Christophe Clement is with the Stockholm University, Sweden (telephone: +4685537 8658, e-mail: clement@fysik.su.se).

Pawel Klimek is with the Stockholm University, Sweden (telephone: +46 85537 8152, e-mail: pawel.klimek@ cern.ch).
The Tile Calorimeter (TileCal) [4] is the hadronic sampling calorimeter of ATLAS in the region $|\eta|<1.7$. The purpose of TileCal is to identify hadronic jets and measure their energy and direction. TileCal also provides vital information for the first level of trigger (L1-trigger), participates in the measurement of the missing energy due to non-interacting particles and to the identification of electrons and photons. It uses steel as an absorber and scintillating plastic tiles as an active material. Several scintillating tiles are grouped together at the read out level to form calorimeter cells. The wavelength shifting fibers couple the light from the tiles to photomultipliers (PMTs), with most TileCal cells being read out by two PMTs, corresponding to two electronic read out channels. The PMT output is a current pulse with its amplitude proportional to the energy deposited in the associated cell. Electronics mounted on the PMT amplifies and shapes the output current pulse. Pulse shaping increases the width at halfmaximum to $50 \mathrm{~ns}$. The analogue pulse is digitized with 7 samples at $25 \mathrm{~ns}$ intervals which are read out upon a trigger accept from the L1 trigger (L1A) and used to compute the pulse amplitude, phase and quality factor. 10-bit analogue to digital converters are used for the digitization.

The presence of collisions every $50 \mathrm{~ns}$ and the relatively large read-out window ( $\pm 75 \mathrm{~ns}$ ) lead to a significant fraction of calorimeter cells receiving energy from more than one bunch crossing within the same read out window and within the same physical calorimeter cell (out-of-time pile-up). Out-oftime pile-up degrades the measurement of the energy deposited in a physical cell. A quality factor is computed online for each event and for each calorimeter channel. It allows to identify calorimeter channels presenting out-of-time pile-up. In presence of a high quality factor value all 7 samples are read out to allow for a dedicated treatment of the pile-up signals offline.

A numerical model has been developed to simulate the TileCal pulse shapes and quality factors with and without outof-time pile-up. The simulated observables are compared with actual proton-proton data in order to validate the model. The model includes electronic noise, time resolution effects and non-ideal pulse shapes. The numerical model allows to predict quality factor distributions thus permitting the optimization of the quality factor online selection criteria, under the constraints of available bandwidth while keeping a reliable out-of-time pile-up detection. 


\section{ENERG Y RECONSTRUCTION AND QUALITY FACTOR DEFINITION}

The goal of the energy reconstruction in TileCal is to compute the energy deposited in a TileCal cell from the number of ADC counts measured in each of the two corresponding read out channels. For each channel, 7 samples at 25 ns spacing are available, these samples are referred to as $S_{i}$ with $i=1-7$ and are in units of ADC counts. In order to maximize the dynamic range, either a low or a high amplification (or gain) is used, depending on the pulse amplitude. The ratio between the low and the high amplification is 64. The high gain is applied to pulses up to about $12 \mathrm{GeV}$, while the low gain is applied for higher energies. One ADC count corresponds approximately to $12 \mathrm{MeV}$ of deposited energy in high gain and about $800 \mathrm{MeV}$ in low gain. The exact correspondence is cell-dependent and requires careful calibration [5].

The energy reconstruction combines the $S_{i}$ to first obtain the amplitude in ADC counts and thereafter applies a calibration constant in $\mathrm{MeV}$ per ADC count. The $S_{i}$ are linearly combined to provide the pulse amplitude $A_{o p t}$, the phase with respect to the $40 \mathrm{MHz}$ clock $t_{o p t}$ and the electronic pedestal $P_{o p t}$, as follows:

$$
\begin{aligned}
A_{\text {opt }} & =\sum_{i=1}^{7} a_{i} \cdot S_{i} \\
t_{\text {opt }} & =\frac{1}{A_{o p t}} \sum_{i=1}^{7} b_{i} \cdot S_{i} \\
P_{\text {opt }} & =\sum_{i=1}^{7} c_{i} \cdot S_{i}
\end{aligned}
$$

The linear coefficients are optimized, using the autocorrelation matrix, to minimize the effect of the noise on the reconstructed quantities. This method is called Optimal Filtering. Prior knowledge of the normalized pulse shape function $g(t)$ is required to determine the constants $a_{i}, b_{i}, c_{i}$. The linear coefficients are functions of the true phase of the pulse $\tau$ with respect to the $40 \mathrm{MHz}$ electronic clock. The pulse shape function as well as the linear constants are stored in a dedicated database. Further information on the use of Optimal Filtering for signal reconstruction in TileCal can be found in Ref. [6].

\section{A. Optimal Filtering Offline}

The Optimal Filtering used in this paper is the same that is used to reconstruct offline the data of the ATLAS TileCal in proton-proton collisions. The constants $a_{i}, b_{i}, c_{i}$ are functions of the actual phase $\tau$ of the pulse which is only approximately known a priori. Therefore the Optimal Filtering is applied offline iteratively with an initial assumed value of the phase $\tau$, equal to the time of the maximum of the $S_{i}$. In later iterations, the phase is taken to be equal to $t_{o p t}$ from the previous iteration. In absence of pile-up the iterative algorithm always converges to the true value of the phase with an accuracy better than $0.5 \mathrm{~ns}$ (the algorithm is iterated until the difference between the input phase and $t_{o p t}$ of Eq. 2. is less than $0.5 \mathrm{~ns}$ or the number of iteration reaches 5) [6].
At the end of the iterative procedure, a quality factor $Q_{o p t}$ is computed to verify that the resulting $A_{o p t}, t_{o p t}$ and $P_{o p t}$ together with the pulse shape $g(t)$ do model the data $S_{i}$ accurately. In case of deviation between the actual shape and the expected shape, then $Q_{o p t}$ takes large values which can be used to detect problems in the reconstruction procedure. The quality factor is defined after convergence as follows:

$$
Q_{o p t}=\sqrt{\sum_{i=1}^{7}\left(S_{i}-A_{o p t} \cdot g_{i}-P_{o p t}\right)^{2}}
$$

where the $g_{i}$ are the values of the normalized pulse shape computed at the time of the 7 samples $S_{i}$.

\section{B. Optimal Filtering Online}

The Optimal Filtering is also run online by the TileCal Digital Signal Processors (DSP) which perform the above linear combinations in real time. Above a trigger rate $50 \mathrm{kHz}$ the Optimal Filtering must be performed without iterations due to insufficient processing time in the DSP. It was also found that in the presence of out-of-time pile-up it is better not to perform the iterations. This is due to the fact that the phase $\tau$ needed to compute $a_{i}, b_{i}, c_{i}$ is known from timing calibration within a few nanoseconds. On the other hand, the presence of out-of-time pile up can lead to $t_{\text {opt }}$ values far from nominal and thus bias the energy reconstruction. For this reason only non-iterative optimal filtering is currently applied online.

\section{PILE-UP SCENARIOS}

The large per bunch crossing luminosity of the LHC leads to a high probability of multiple proton-proton interactions in the same bunch crossing. This leads to in-time energy deposits from multiple collisions in the same TileCal cell from the same bunch crossing from different $p$-p collisions. We refer to this type of pile-up as "in-time pile-up" and it can be addressed by determining its average effect on the measured calorimeter energies. It is not discussed further in this paper.

The second type of pile-up, or "out-of-time" pile-up arises when bunch crossings are close in time, and that the signal shaping time is larger than the time between consecutive bunches. In the case of TileCal, the long signal shaping time requires a read-out window of \pm 75 ns around a triggered event, to be compared with a bunch spacing of $50 \mathrm{~ns}$ during the 2011 data-taking. The pulse shape is adjusted in such a way that the maximum of the pulse is located close to the fourth sample, $S_{4}$. The "out-of-time" pile-up results in the superposition of pulses shifted in time resulting in anomalous pulse shapes which can be detected thanks to large values of $Q_{\text {opt }}$. In 2011 the LHC bunch spacing was $50 \mathrm{~ns}$ and therefore there are two possible pileup scenarios: the pile-up signal results from energy deposited $-50 \mathrm{~ns}$ from the collision of interest; the pile-up signal results from energy deposited +50 ns from the collision of interest. Due to the TileCal pulse shape these have different effects on the reconstructed signal in the collision of interest. Both scenarios are studied and the expected quality factor distributions are computed in both scenarios. Fig. 1 shows an illustration of an out-of-time pile-up 


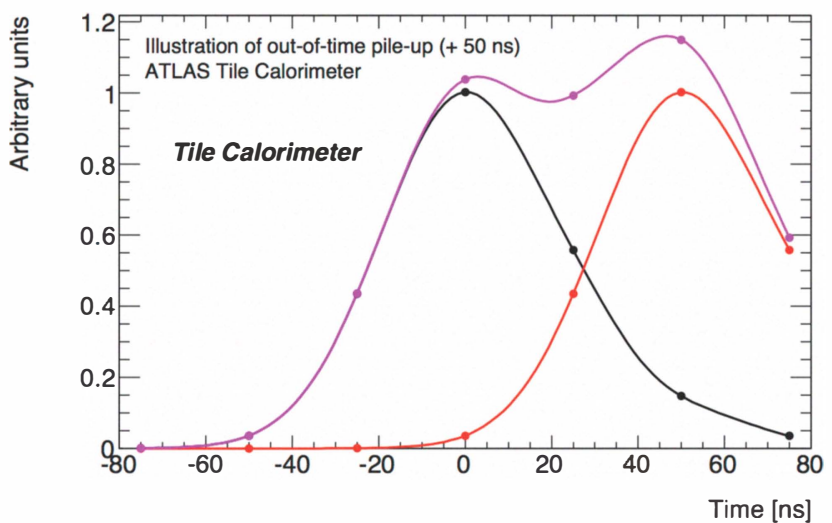

Fig. 1. Illustration of the superposition of an in-time pulse with an out-of-time pile-up pulse of equal amplitude at $+50 \mathrm{~ns}$ in the ATLAS Tile Calorimeter. The pulse shapes shown here are approximate functional parameterizations of actual pulse shapes, but are not actually used either in the energy reconstruction, nor in the pile-up simulation.

pulse at $+50 \mathrm{~ns}$, in the case where the in-time and out-of-time pulses have the same amplitude.

\section{Pulse Shape Simulator}

\section{A. Working Principle}

The pulse shape simulator is based on pseudo-random number generators to generate the 7 samples $S_{i}$ which constitute a digitized pulse. The pulse simulator uses a number of input probability density functions that model the electronic noise, the distribution of random pulse phases, the timing resolution and the distribution of pulse width. All these components are required to reproduce the characteristics of the digitized pulse in data. The most significant parameters of the model are the noise and the variations in pulse widths which are both adjusted such that the predicted $Q_{o p t}$ in the simulator agrees with the $Q_{o p t}$ distribution measured in data in absence of outof-time pile-up. Thanks to the iterative procedure, the value of $Q_{o p t}$ is found to be rather independent of the timing effects. Later on in Section $\mathrm{V}$ the pulse simulator is used to derive the expected $Q_{F}$ distribution for out-of-time pulses.

\section{B. Input to the Model}

The parameters of the model are the following.

1) Pulse shape: As shown in Eq. 4 the quality factor is a measure of the difference between the ideal pulse shape used to derive the optimal filtering coefficients and the actual pulse shapes in the real detector. It is shown on Fig. 2 that the pulse shapes in TileCal are consistent with the ideal pulse shapes. Nevertheless even small pulse shape differences will be enlarged by signal amplitudes.

The normalized ideal pulse shape used in the optimal filtering is denoted $g(t)$, or $g_{i}$ at the times of the $S_{i}$ where the pulse is sampled. The function $h(t)$ denotes the normalized real pulse shape in an actual TileCal channel. One can thus write $h(t)=g(t)+\delta(t)$ or $h_{i}=g_{i}+\delta_{i}$ at the times of the samples $S_{i}$, where $\delta$ quantifies the deviation between the ideal pulse shape and the actual pulse shape in the detector. In this

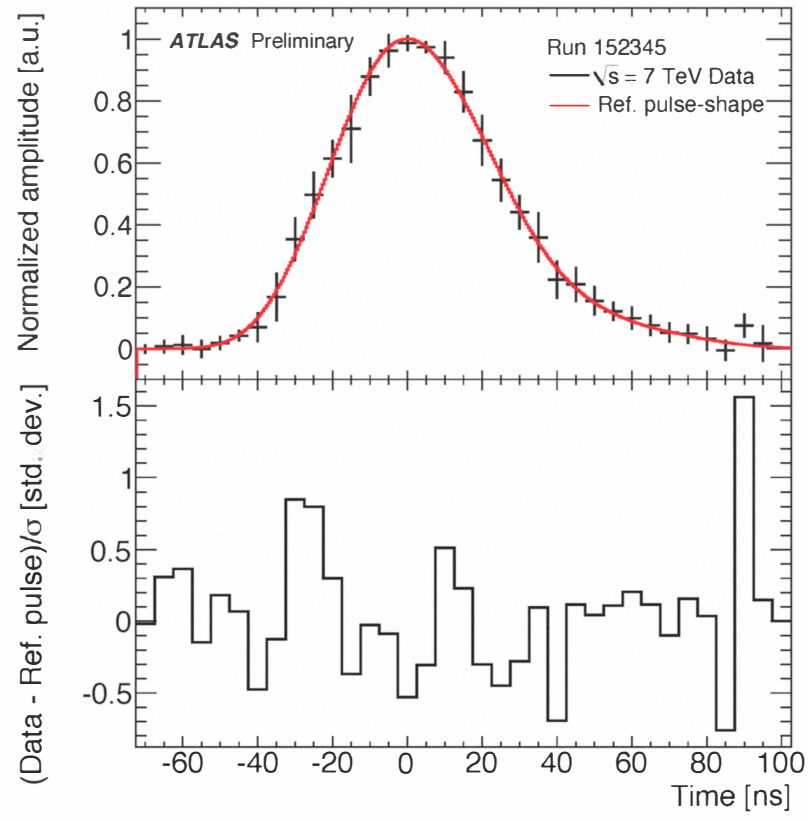

Fig. 2. Pulse shape reconstructed with the Optimal Filtering averaged over all good channels and full energy range in high gain in the TileCal from data 2010. Overlaid in red is the reference high gain pulse shape used for reconstruction. Bottom: The deviation between data and reference pulses in units of standard deviations. $\sigma$ is the statistical spread on the data.

case one can write $S_{i}=A \cdot h_{i}+P=A \cdot g_{i}+A \cdot \delta_{i}+P$, where $P$ is the actual pedestal and $A$ is the actual amplitude. Thus the quality factor of Eq. 4 can be reexpressed as:

$$
Q_{o p t}=\sqrt{\sum_{i=1}^{7}\left(A g_{i}+\delta_{i}+P-A_{o p t} \cdot g_{i}-P_{o p t}\right)^{2}}
$$

In absence of noise the amplitude and pedestal are perfectly reconstructed by the optimal filtering, thus $P_{o p t}=P$ and $A_{\text {opt }}=A$, which is approximately true at large amplitudes where the electronic noise becomes negligible, the equation above simplifies to

$$
Q_{o p t}=A \cdot \sqrt{\sum_{i=1}^{7}\left(\delta_{i}\right)^{2}}
$$

Therefore at large signal amplitudes the quality factor depends linearly upon the amplitude of the pulse and the slope depends on the difference between the ideal pulse shape and the actual pulse shape in the detector. Fig. 3 shows $Q_{o p t}$ as function of the pulse amplitude in collision data, in absence of out-of-time pile-up, for channels with signals larger than 200 ADC counts, the dependence of the quality factor on the energy appears clearly. For comparison, Fig. 4 shows the quality factor in the simulator if we assume that the measured $S_{i}$ follow the ideal pulse shape. In order to reproduce the quality factor observed in data, the simulator must use a pulse shape that is different from the ideal pulse shape. The pulse shapes in data are modeled by the normalized ideal pulse shapes, with a modified width. Widened or narrowed pulses are obtained by using a new pulse shape given by $g(\alpha t)$, where $g$ is the 


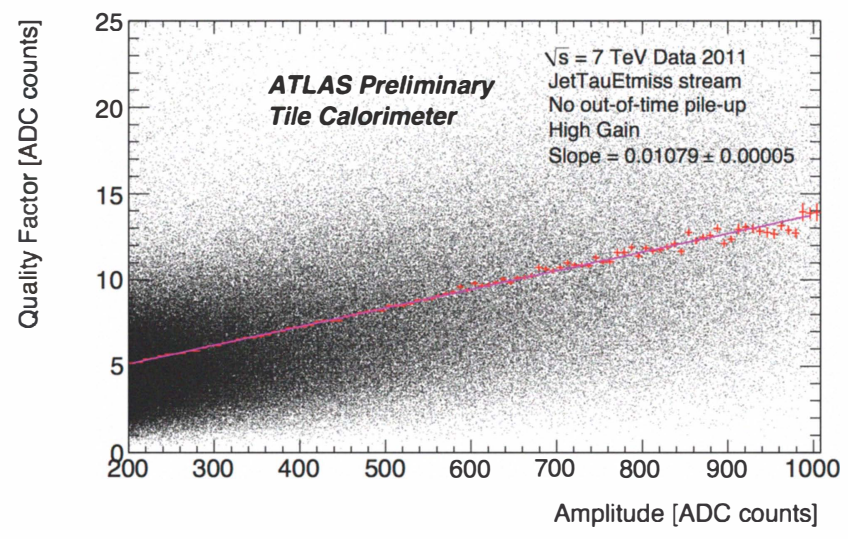

Fig. 3. Quality factor as a function of the reconstructed amplitude $A_{o p t}$ in Data 2011. This data is in high gain and was recorded while the LHC was running with only 2 bunches per beam separated by at least $2.5 \mu$ s. The $\mathrm{x}$-axis shows the amplitude in ADC counts. One ADC count corresponds approximately to $12 \mathrm{MeV}$.

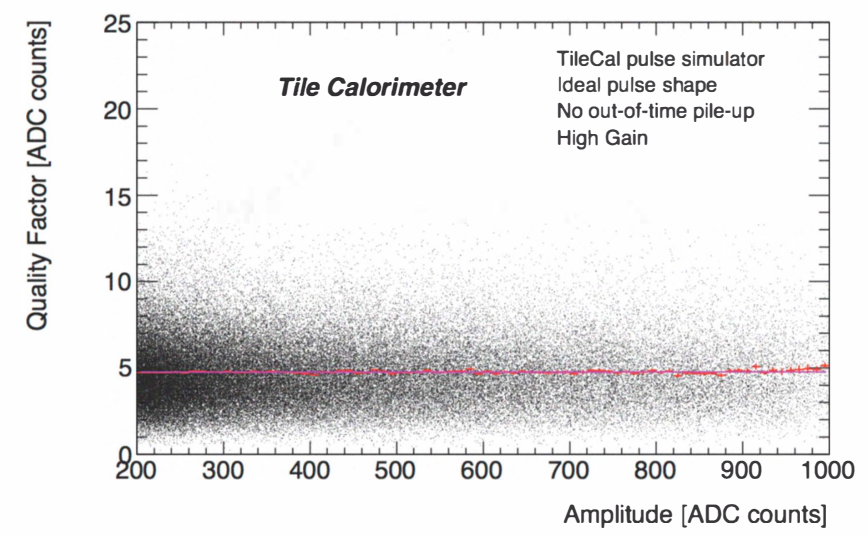

Fig. 4. Quality factor as a function of the reconstructed amplitude $A_{o p t}$ in the TileCal pulse simulator with ideal pulse shape, but with timing and noise effects emulated. No out-of-time pile-up is present here. The $\mathrm{x}$-axis shows the amplitude in ADC counts before cell-dependent calibration constants are applied. One ADC count corresponds to approximately $12 \mathrm{MeV}$.

ideal pulse shape used earlier and $\alpha$ is a factor close to one. A value of $\alpha$ equals to one gives the ideal pulse shape, while $\alpha<1$ corresponds to a narrower pulse and $\alpha>1$ corresponds to a wider pulse. The $\alpha$ factor is taken to follow a Gaussian distribution with a mean value of 1 and a standard deviation $\sigma$ that is adjusted so that the quality factor distribution observed in the simulator matches that of the data.

2) Energy distribution: As the quality factor is dependent on the amplitude, the simulator has to use the same energy distribution as the data. The pulse shape simulator is validated by comparing its result with the data in Section IV-C using TileCal data collected with a jet or missing energy trigger. For this comparison the probability density function of the energy measured in the TileCal cells is extracted from the data and used to generate the amplitude of the pulses in the simulator. In Section $\mathrm{V}$ it is shown that the energy distribution in TileCal cells can be extracted without bias due to the trigger, in order to model the amplitude distribution of the out-of-time pulses.

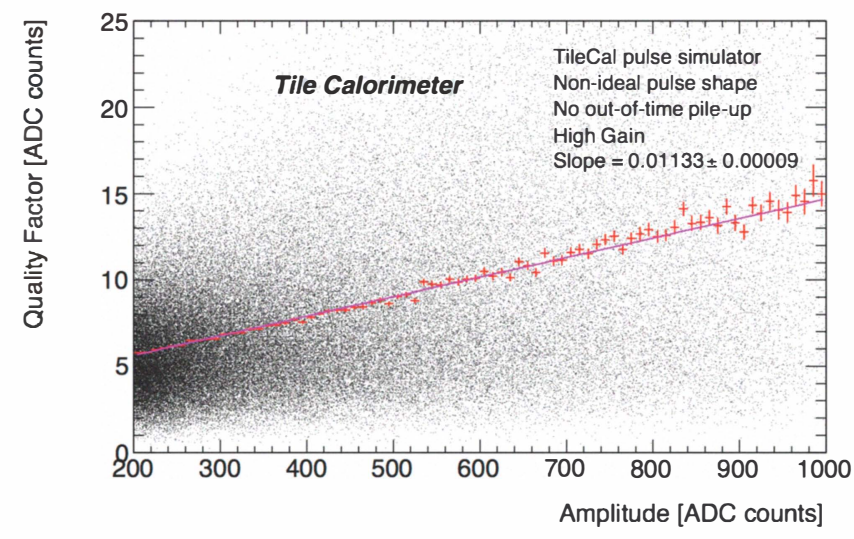

Fig. 5. Quality factor as a function of the reconstructed amplitude $A_{o p t}$ in TileCal pulse simulator with non-ideal pulse shapes, and with timing and noise effects emulated (full model of the pulse simulator). No out-of-time pile-up is present here. The $\mathrm{x}$-axis shows the amplitude in ADC counts before cell-dependent calibration constants are applied. One ADC count corresponds approximately to $12 \mathrm{MeV}$.

3) Channel to channel phase variation $\phi_{c h}$ : Ideally the peak of the signal pulses should be perfectly centered in the middle of \pm 75 ns read-out window. In the actual Tile Calorimeter the position of the pulse peak has been shown to be within $3 \mathrm{~ns}$ of the middle of the read-out window [4]. This effect is taken into account in the simulator by randomly offsetting the simulated pulses before reconstruction with a random phase that is Gaussian distributed with a mean of zero and a standard deviation of $3 \mathrm{~ns}$.

4) Time resolution $\phi_{\text {res }}$ : The precision of the time $t_{\text {opt }}$ determined with the Optimal Filtering is a known function of the pulse amplitude and has been determined in data. The resolution on $t_{\text {opt }}$ propagates to the quality factor through equation 2 and is therefore taken into account in the simulator by randomly shifting the time of the pulse by an amount determined from a Gaussian with mean zero and with an energy dependent standard deviation, given by:

$$
\sigma_{T}=\sqrt{p_{0}^{2}+\left(\frac{p_{1}^{2}}{\sqrt{E}}\right)^{2}+\left(\frac{p_{2}^{2}}{E}\right)^{2}}
$$

where the parameters $p_{0}=0.82 n s, p_{1}=0 n s \cdot \mathrm{GeV}^{1 / 2}$ and $p_{2}=2.30 \mathrm{~ns} \cdot \mathrm{GeV}$ have been determined on data.

5) Incoherent electronic noise: The incoherent electronic noise modifies the measured values of the samples $S_{i}$ randomly around the normalized pulse shape, the effect is to first approximation uncorrelated between the samples $S_{i}$. This is the second most significant contribution to the quality factor, after the pulse shape, but becomes the dominant factor at low amplitudes. The tail of high quality factor values is particularly sensitive to the noise. For this reason the simulator uses the double Gaussian noise model that was found to describe the TileCal noise data [4]. The noise constants used to smear the $S_{i}$ were adjusted so that the quality factor distribution obtained with the simulator reproduces the quality factor in the data. 


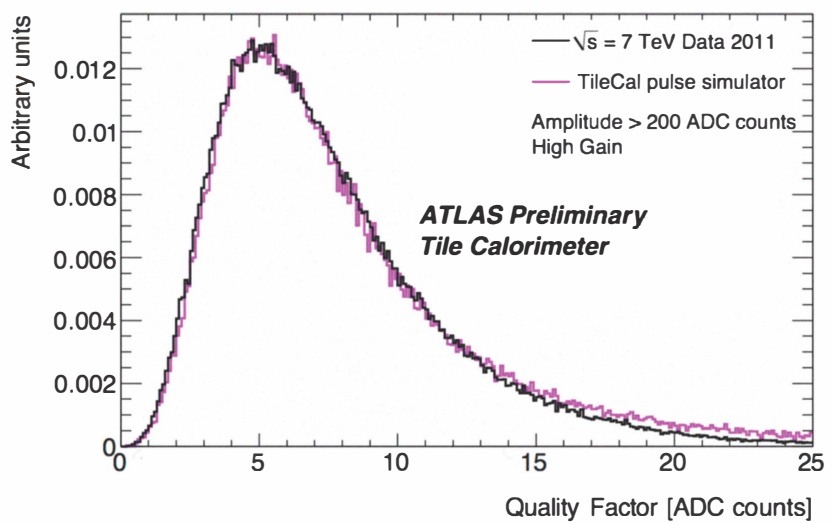

Fig. 6. Comparison of the quality factor distribution in data taken in March 2011 (black) and with the pulse simulator with non-ideal pulse shapes (purple) for pulses with amplitudes above 200 ADC counts. This data was recorded while the LHC was running with only 2 bunches per beam separated by at least $2.5 \mu \mathrm{s}$.

\section{Comparison of the Quality Factor in Data and TileCal Pulse Simulator}

The quality factor distribution is computed in data using an integrated luminosity of $60 \mathrm{nb}^{-1}$ taken in March 2011 at a period where the LHC was operating with only 2 bunches per beam, separated by at least $2.5 \mu \mathrm{s}$, therefore ensuring the absence of out-of-time pile-up. The collisions were selected to pass either a calorimeter trigger or a missing energy trigger. Fig. 3 shows the energy dependence of the quality factor in this data set. Fig. 5 shows the corresponding distribution of quality factor $Q_{o p t}$ as function of $A_{o p t}$ obtained in the pulse simulator, in absence of out-of-time pile-up as for the data of Fig. 3 . The resulting quality factor distribution in data and from the simulator is shown in Fig. 6 and shows a fair agreement apart from the high tail of the quality factor distribution. This demonstrates that the pulse shapes can be simulated in such a way that a complex quantity such as the quality factor can be reproduced. The simulator can then be used to predict the quality factor distribution in presence of out-of-time pile-up in order to derive the optimal criteria to detect out-of-time pile-up while keeping the amount of read out data within the bandwidth budget of TileCal.

\section{Quality Factor Simulation with Pile-uP}

\section{A. Amplitude of Out-of-Time Pulses}

The average signal amplitude for in-time pulses is related to the trigger criteria used to record the event, since for instance requiring several highly energetic hadronic jets will certainly increase the amount of energy deposited in the calorimeter and hence the likelihood that a calorimeter channel received a large signal.

The out-of-time pulses on the other hand belong to collisions that did not pass the trigger. They are recorded by chance since they were close in time to a collision that passed the trigger. Therefore the energy distribution from pileup is that from unbiased collisions before the trigger. This energy distribution can be extracted from data by using a

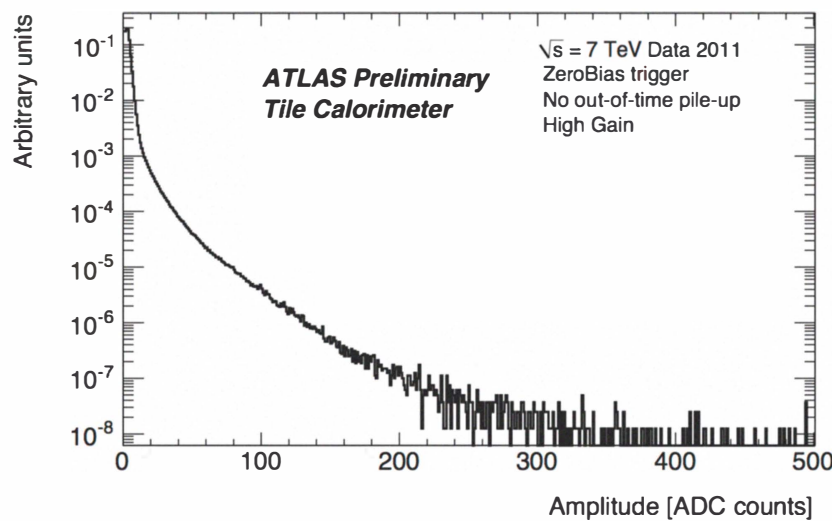

Fig. 7. Amplitude distribution of out of time pulse. Parameterization extracted from zero bias 2011 data in high gain in absence of out-of-time pile-up. This data was recorded while the LHC was running with only 2 bunches per beam separated by at least $2.5 \mu \mathrm{s}$. The $\mathrm{x}$-axis shows the amplitude in ADC counts before cell-dependent calibration constants are applied. The calibration factor is approximately $12 \mathrm{MeV}$ per ADC count.

specific trigger. ATLAS possesses a so-called a zero bias trigger, which records a small fraction of collisions randomly selected in coincidence with the crossing of two populated proton bunches. This zero bias trigger allows to measure the energy distribution in TileCal channels without the effect of the trigger bias, and is therefore used as a model to extract the probability density function of the amplitude of the outof-time pulses. This amplitude distribution is extracted from no pile-up ATLAS data from March 2011 at a time where the LHC was operating with only two bunches per beam, separated with at least $2.5 \mu \mathrm{s}$, the corresponding distribution is shown in Fig. 7. This amplitude distribution is used as probability density function to generate out-of-time pulses in the pulse simulator and compute the quality factor in presence of pileup in Section V-B.

\section{B. Quality Factor Distributions in Presence of Out-of-Time Pile-up}

The effect of the out-of-time pile-up on the quality factor is first studied in absence of noise, or timing effects and for ideal pulse-shapes. In this simplified model one can study the effect of the relative sizes of the in-time and out-of-time pulses. Fig. 8 shows the dependence of the quality factor $Q_{o p t}$ as a function of the in-time pulse amplitude $A_{\text {in }}$ given on the xaxis and for different values of the ratio between the in-time and out-of-time pulse amplitude $A_{\text {out }}$. It shows two important features, first that for a given ratio of $A_{\text {out }} / A_{\text {in }}$, the quality factor increases linearly with the amplitude of the in-time pulse, and second that the dependence on the amplitude $A_{\text {in }}$ gets steeper when the ratio $A_{\text {out }} / A_{\text {in }}$ gets closer to one. The worst case scenario occurs for in-time and out-of-time pulses of equal amplitude, in that case the quality factor becomes maximal. The introduction of an out-of-time pile-up pulse is equivalent to introducing a deviation between the ideal pulse shape and the real pulse shape as discussed in Sect. IV-B1. The linear dependence on the amplitude observed here is therefore 


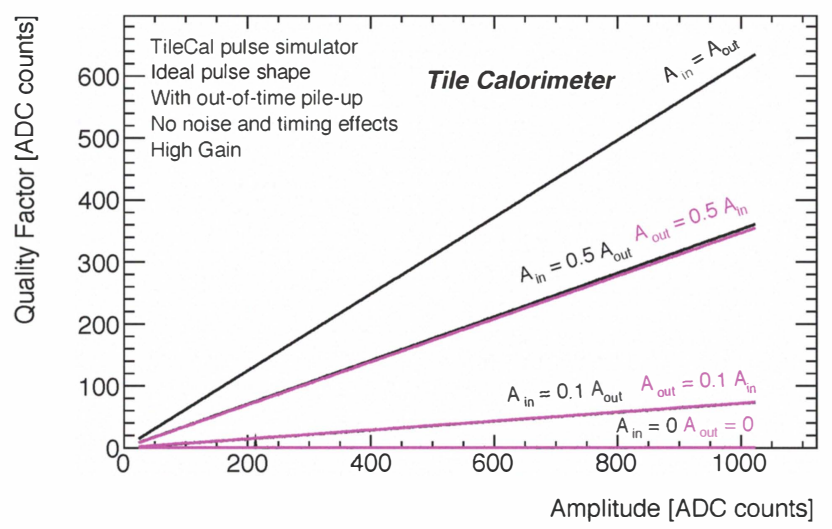

Fig. 8. Quality factor as a function of the amplitude in different pile-up scenarios in the TileCal pulse simulator with ideal pulse shape, in absence of noise or timing effects. The amplitude $A_{i n}\left(A_{\text {out }}\right)$ is the amplitude of the in-time (out-of-time). The $\mathrm{x}$-axis represents the amplitude of the in-time pulse and is given in ADC counts, before cell-dependent calibration constants are applied. One ADC count corresponds to approximately $12 \mathrm{MeV}$.

consistent with the observation of a linear dependence upon pulse amplitude made in Section. IV-B1.

In order to compute a realistic distribution of the quality factor in presence of out-of-time pile-up, the amplitude of the out-of-time pulse is modeled with the full model, and the probability density function obtained using the zero-bias data as described in Sec. IV. Fig. 9 compares the quality factor in absence (black) and in presence of out-of-time pile-up (red and purple) obtained for this model and for an in-time pulse of 1000 ADC counts, corresponding to about $12 \mathrm{GeV}$. Only the out-of-time pulses yielding an effect of more than $1 \%$ bias on the reconstructed amplitude have been retained for this figure, which occurs only for approximately 1 in $O\left(10^{3}\right)$ channels per bunch crossing with an expected average number of collisions of $\langle\mu\rangle=3.3$. It illustrates that for significant out-of-time pile-up pulses the distribution of quality factor is quite different in case of out-of-time compared to no outof-time pile-up. There is a clear separation between the two cases. As it has been shown that the quality factor is linearly increasing with the amplitude of the pulses, further separation can be achieved by dividing the quality factor given in Fig. 9 and Eq. 4.

\section{CONCLUSIONS}

A numerical model of the ATLAS Tile Calorimeter pulses has been developed in the form of a pulse simulator. This model takes into account small variations in signal pulse shapes, timing resolution and small timing miscalibration effects and uses a realistic model of the calorimeter noise. The simulator is shown to be able to reproduce the quality factor distributions in collisions in absence of out-of-time pile-up for pulses with amplitudes larger than 200 ADC counts. The model is still in development and will be improved to model the data quality factor for the full range of pulse amplitude. The signal amplitude for TileCal channels is measured in zero bias triggered data and used as a model for pulses of from outof-time pile-up collisions. Using this model of the data, the

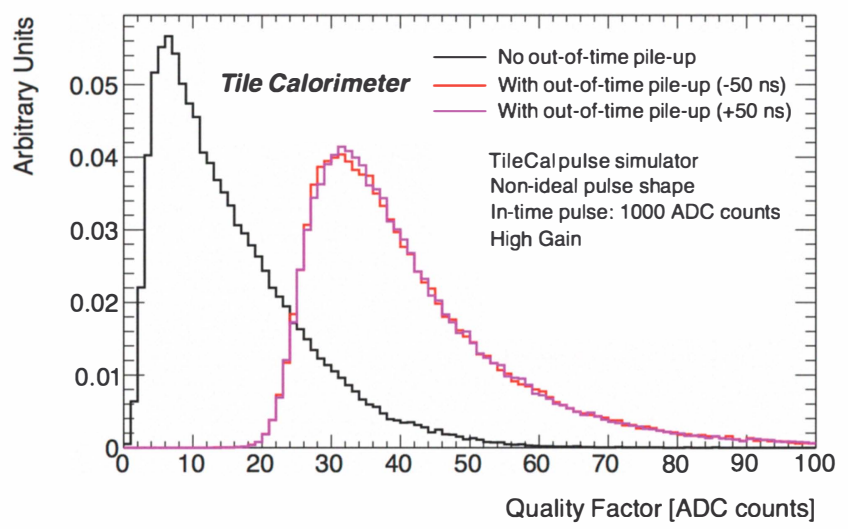

Fig. 9. Normalized distributions of quality factors in TileCal simulator with non-ideal pulse shapes for an in-time pulse of 1000 ADC counts (approximately $12 \mathrm{GeV}$ ), without (black) and with out-of-time pile-up (red and purple). The amplitude of the out-of-time pulse follows the distribution in the zero bias trigger and requiring additionally that the effect of the out-oftime pulse on the reconstructed amplitude is at least $1 \%$. The two out-of-time pile-up scenarios with $-50 \mathrm{~ns}$ (red) and $+50 \mathrm{~ns}$ (purple) are shown.

distribution of quality factor in the presence of out-of-time pile-up is calculated. It shows that significant discrimination can be achieved thanks to the the quality factor between the presence and the absence of out-of-time pile-up in case the amplitude of the out-of-time pile-up large enough to affect the amplitude measurement. Using the predicted distributions of quality factor with and without out-of-time pile, the presence of significant out-of-time pile-up can be identified and a specific treatment of the double pulses can be performed.

\section{REFERENCES}

[1] ATLAS Collaboration, The ATLAS Experiment at the Large Hadron Collider, 2008 JINST 3 S08003.

[2] P. W. Higgs, Broken symmetries and the masses of gauge bosons, Phys. Rev. Lett. 13 (1964), 508-509.

[3] H. Baer, X. Tata, Weak Scale Supersymmetry, DOI: 10.2277/ 0521857864, ISBN:9780521857864 (2006) and references therein.

[4] ATLAS Collaboration, Readiness of the ATLAS Tile Calorimeter for LHC Collisions, CERN-PH-EP-2010-024. Eur.Phys.J. C70 (2010) 1193-1236.

[5] J. Carvalho et al. (on behalf of the ATLAS Collaboration), The TileCal/ATLAS calorimeter calibration systems, to be published in proceedings of ICATPP 2011, ATL-TILECAL-PROC-2011-011 (2011).

[6] A. Valero, Implementation and performance of the signal reconstruction in the ATLAS Hadronic Tile Calorimeter, to be published in proceedings of TIPP 2011, ATL-TILECAL-PROC-2011-008 (2011). 